\title{
Influenza: overview on prevention and therapy
}

\section{SUMMARY}

Quadrivalent influenza vaccination is recommended annually for adults and children aged six months to 64 years.

High-dose or adjuvanted trivalent vaccines are recommended annually for people 65 years and over.

If started early enough, neuraminidase inhibitors reduce symptom duration by approximately one day. Treatment should be considered in patients with severe disease requiring hospitalisation or who are at risk of complications.

Chemoprophylaxis is not a substitute for vaccination but can be considered in high-risk individuals with an inadequate or ineffective vaccination status.

\section{Introduction}

Influenza causes considerable morbidity and mortality in Australia each year. Routine vaccination is the most important intervention for preventing illness and severe complications. Hand hygiene, cough etiquette and voluntary home isolation are also important factors in reducing transmission. The mainstay of treatment is symptom control and management of secondary complications. However, a number of antiviral drugs are available to treat influenza. They also have a small role in prophylaxis.

\section{Preventing influenza}

Influenza is a viral infection, mainly of the respiratory tract. There are two influenza A subtypes circulating in humans - A/H1N1 and A/H3N2 - and two influenza B subtypes - the Yamagata and Victoria lineages.

Annual vaccination is recommended for all individuals above six months of age (with the exception of any patients who have previously experienced anaphylaxis to the influenza vaccine or one of its components). Annual influenza vaccination is funded under the National Immunisation Program for people at increased risk of influenza morbidity and mortality. This includes the following:

- those over six months of age with medical risk factors

- Aboriginal and Torres Strait Islanders aged six months and over

- all Australians aged over 65 years

- pregnant women.

Influenza in pregnancy is associated with an increased risk of maternal morbidity and mortality, along with preterm delivery. The influenza vaccine can be administered at any stage of pregnancy.

It is particularly important that healthcare providers in hospitals and general practices are vaccinated, given their likely exposure to individuals with influenza. This mitigates their potential for transmitting the virus, especially to people at risk of complications.

The antibody response to the vaccine takes approximately two weeks, with a period of optimal vaccine efficacy of around four months post vaccination. In Australia the seasonal influenza vaccine becomes available in March or April, and this is an appropriate time to vaccinate. The existence of several subtypes, along with seasonal antigenic changes, makes it difficult to predict which influenza strain will cause the most substantial burden of disease each year.

Given that the vaccine formulation is determined nine months before the influenza season, the strains included are based on the previous winter's circulating viruses, and are an informed prediction of what will be most prevalent in the coming season. Vaccine efficacy is variable from year to year and in different populations because of this.

\section{Trivalent and quadrivalent vaccines}

There is a range of different influenza vaccines available in Australia ${ }^{-7}$, some of which are provided through the National Immunisation Program (see Table). ${ }^{8}$ Trivalent vaccines cover the two influenza A types and a single $B$ lineage whereas the quadrivalent vaccines cover the additional $B$ virus lineage. There is evidence in the transition from trivalent to quadrivalent vaccines that the

\section{Christopher Robson \\ Sai Rupa Baskar Robert Booy ${ }^{2,3}$ \\ Patricia E Ferguson ${ }^{1,3}$ Nicole Gilroy ${ }^{1}$ Jen Kok ${ }^{4}$ Indy Sandaradura, ${ }^{1,3}$ Dominic Dwyer ${ }^{1,3,4}$}

'Westmead Hospital, Sydney

2 Kids Research Institute at The Children's Hospital at Westmead, Sydney ${ }^{3}$ University of Sydney ${ }^{4}$ NSW Health Pathology, Sydney

Full author details are available online.

\section{Keywords} antivirals,

chemoprophylaxis, influenza, vaccines

Aust Prescr 2019;42:51-5 https://doi.org/10.18773/ austprescr.2019.013

Corrected 3 June 2019

This is the corrected version of the article.

Correction notice available at: https://doi.org/10.18773/ austprescr.2019.045 


\section{Table 2019 Australian seasonal influenza vaccines available on the National Immunisation Program}

\begin{tabular}{|c|c|c|c|}
\hline Type of vaccine & Age group & Brand name & Efficacy in clinical trials \\
\hline \multirow[t]{4}{*}{ Quadrivalent } & 6-35 months & FluQuadri Junior & \multirow{2}{*}{$\begin{array}{l}\text { The quadrivalent vaccines elicited non-inferior antibody responses to all A strains ano } \\
\text { corresponding B strains compared to a trivalent vaccine. Superior immunogenicity } \\
\text { was shown for non-corresponding B strains in the quadrivalent vaccine. }\end{array}$} \\
\hline & 3 years and over & FluQuadri & \\
\hline & 3 years and over & Fluarix Tetra & $\begin{array}{l}\text { Fluarix Tetra elicited non-inferior antibody responses compared to the Fluarix } \\
\text { trivalent vaccine and superior responses for the additional B strain not in the } \\
\text { trivalent comparator. }{ }^{2}\end{array}$ \\
\hline & 5 years and over & Afluria Quad & $\begin{array}{l}\text { Afluria Quad elicited non-inferior immune responses to all comparator strains when } \\
\text { compared to two trivalent vaccines containing alternate B strains. Superior immune } \\
\text { responses to the trivalent unmatched strains were found. }{ }^{4}\end{array}$ \\
\hline Trivalent & 65 years and over & $\begin{array}{l}\text { Fluad (MF59- } \\
\text { adjuvant) }\end{array}$ & $\begin{array}{l}\text { Fluad (MF59-adjuvant) elicited significantly higher antibody responses compared } \\
\text { to a non-adjuvanted trivalent vaccine in older people, including those with } \\
\text { underlying medical conditions. Significantly higher responses were observed } \\
\text { against heterologous A strains, and higher antibody responses were observed } \\
\text { for H3N2 strains up to } 12 \text { months after vaccination. }{ }^{6} \text { A systematic review found } \\
\text { that Fluad (MF59-adjuvant) was more effective than non-adjuvanted trivalent } \\
\text { vaccine in preventing hospitalisation from pneumonia/influenza in older people } \\
\text { (51\%, } 95 \% \text { confidence interval } 39-61 \%) .7\end{array}$ \\
\hline
\end{tabular}

quadrivalent vaccines confer improved protection without any obvious increase in adverse reactions. ${ }^{9}$ The standard influenza vaccination for children and adults, including pregnant women, is now a single quadrivalent preparation. An exception to this is children aged six months to nine years who are receiving the vaccine for the first time, and those in the first year after receiving a solid organ or haematopoietic stem cell transplant. These patients should receive two doses at least four weeks apart to induce an optimal immune response.

\section{High-dose and adjuvanted trivalent vaccines for older adults}

People aged 65 and over have an increased risk of not only contracting influenza, but also of developing serious complications including heart attack, decompensated cardiac failure, pneumonia and death. ${ }^{10,11}$

Two new trivalent vaccines for this age group were introduced in 2018, and one (Fluad) will be funded on the National Immunisation Program in 2019. ${ }^{8}$ They are currently not recommended for younger individuals, however recent literature suggests that recipients of solid organ transplants may also benefit from these vaccines. ${ }^{12}$

The high-dose preparation (Fluzone High-Dose) contains 60 microgram of the haemagglutinin antigen, which is four times more than the antigen content of other vaccine formulations for those under 65 years old. The adjuvanted vaccine (Fluad) contains MF-59, a squalene-based emulsion that can rapidly induce antigen-specific CD4 responses. This results in strong and lasting T- and B-cell memory immune responses. The advantage of adjuvanted vaccines is they induce a broad host response while at the same time being dose sparing.

Recent studies have shown that administering trivalent flu vaccines, either at higher dose or with an adjuvant to increase immunogenicity, improves vaccine efficacy in people aged 65 or more..$^{5,13-15}$ Other benefits include reduced hospitalisation for influenza and its complications, and reduced influenza-related deaths. ${ }^{13,16}$ A significantly higher rate of injection-site reactions has been reported with these preparations compared to standard trivalent vaccines (approximately $30 \%$ vs $20 \%$ of recipients). However, there has been no observed difference in the rate of serious adverse events. ${ }^{17,18}$

The absence of the additional $B$ lineage in these trivalent vaccines is not thought to be of notable detriment in older people for a number of reasons. First, the influenza A subtype A/H3N2 is likely to be responsible for the bulk of infections and serious complications in older patients. Second, a meta-analysis of several studies found that vaccination against a single influenza B strain confers up to $50 \%$ crossprotection against mismatched influenza $B$ strains. ${ }^{19}$

At this stage, there have been no head-to-head trials comparing high-dose or adjuvanted trivalent vaccines with quadrivalent vaccination. New vaccine preparations (for example, using nanoparticles to carry influenza antigens) are under trial.

To date the safety and efficacy of adjuvanted or high-dose antigen influenza vaccines in pregnancy has not been established. Age-specific vaccine is recommended for pregnant women. 


\section{Antiviral drugs}

There are two main classes of antiviral drugs that have been used for the treatment and prophylaxis of influenza - neuraminidase inhibitors and adamantanes. Multiple novel therapies are currently in development.

\section{Neuraminidase inhibitors}

Neuraminidase inhibitors are the mainstay of antiviral therapy against influenza. However, they need to be started within 48 hours of symptom onset and are most effective within 24 hours.

They inhibit the viral neuraminidase enzyme, preventing the virus from escaping the host cell. ${ }^{20}$ Three neuraminidase inhibitors are currently registered in Australia - oral oseltamivir, inhaled zanamivir and intravenous peramivir. ${ }^{21}$

\section{Efficacy}

Oseltamivir shortens the duration of symptoms in uncomplicated influenza by approximately one day. ${ }^{22,23}$ The majority of studies were in healthy adults, and this effect has not been shown in asthmatic children. ${ }^{22,23}$ Inhaled zanamivir has shown a similar reduction in duration of symptoms in adults but has no significant effect in children. ${ }^{22,23}$ Single-dose intravenous peramivir is non-inferior to oseltamivir in adults and is a potential alternative for those who cannot take oral or inhaled medicines. ${ }^{24}$

A newer long-acting neuraminidase inhibitor, laninamivir, achieves high concentrations in lung tissue with the potential to treat influenza following a single inhaled dose. It has comparable efficacy to oseltamivir in adults. ${ }^{25,26}$ An intravenous form of zanamivir has been recently studied in populations with severe influenza and also shows similar outcomes to oseltamivir. ${ }^{27}$ While these two drugs are not currently registered in Australia, intravenous zanamivir has been used through the Special Access Scheme for critically ill patients with influenza. ${ }^{21}$

The role of neuraminidase inhibitors in reducing influenza complications is less clear. ${ }^{22,23}$ While oseltamivir has been shown to reduce unverified pneumonia, this has not been confirmed in trials with robust diagnostic criteria. ${ }^{23}$ It has also not been shown to reduce the rate of hospital admissions. ${ }^{22}$

Zanamivir has not been found to reduce pneumonia complications and its effect on hospital admissions has not been studied. ${ }^{22,23}$ In patients with influenza the use of neuraminidase inhibitors has been associated with a mortality benefit, with delayed treatment resulting in increased mortality. ${ }^{28}$

\section{Recommendations for treatment}

Prompt commencement of neuraminidase inhibitors is recommended for patients with confirmed or suspected influenza who require hospitalisation, or are at risk of complications (including children $<5$ years, adults $\geq 65$ years, pregnant women, immunosuppressed patients or significant comorbidities), or have severe, complicated or progressive disease. ${ }^{29-31}$ Therapy should begin within 48 hours of the onset of illness, but in severe disease treatment may still be beneficial if given outside this timeframe. ${ }^{28,30}$ Treatment should also be considered in those who have household contacts who are at high risk of influenza complications. ${ }^{30,31}$ The recommended duration of therapy (oseltamivir and zanamivir) is five days. ${ }^{29}$ In healthy outpatients with uncomplicated influenza, treatment can be of limited benefit. ${ }^{29,30}$ Antibiotics are only indicated when patients have bacterial complications. ${ }^{29,31}$

\section{Antiviral resistance}

Antiviral resistance has been well described in at-risk populations including immunocompromised hosts and young children due to a high virus burden and prolonged replication promoting resistance mutations. ${ }^{32}$ Factors that increase the risk of resistance include suboptimal antiviral dosing and cross-transmission of resistant strains in outbreaks. ${ }^{32,33}$ The $\mathrm{H} 275$ Y mutation is commonly associated with oseltamivir-resistant influenza A strains, but laninamivir and zanamivir rarely show cross-resistance to strains expressing this mutation. ${ }^{32}$

\section{Adamantanes}

Adamantanes work by inhibiting the M2 ion channel. ${ }^{20}$ They are not recommended due to widespread resistance in circulating influenza viruses. ${ }^{20,29}$ Currently amantadine is the only drug to be registered in Australia for influenza. Its use is limited to prophylaxis of influenza A. ${ }^{21,34}$ When used for treatment, amantadine shortens the duration of fever by approximately one day, but has no effect on nasal shedding or upper airways viral clearance. ${ }^{34}$

\section{Antiviral prophylaxis}

There is a role for neuraminidase inhibitors in prophylaxis. Oseltamivir and zanamivir are approved in Australia for this and have shown to significantly reduce the risk of symptomatic influenza. 22,23 However, chemoprophylaxis should not be considered as an alternative to vaccination. ${ }^{29}$

For individual benefit, post-exposure prophylaxis with neuraminidase inhibitors should be considered for contacts who are at high risk of influenza complications and cannot be (or have not been) vaccinated or are 
likely to have an inadequate or ineffective vaccine response..$^{30}$ In household settings, chemoprophylaxis can be considered for remaining contacts of a suspected or confirmed influenza case, if there is another member at high risk of influenza complications. ${ }^{30}$

During an influenza outbreak in residential care facilities (including aged care, correctional facilities, hostels), antiviral prophylaxis should only be considered in addition to other infection control measures. The decision to administer antivirals must be made in collaboration with treating doctors, public health authorities and the local outbreak management team. ${ }^{35}$ When used, antiviral prophylaxis should be started within 24 hours of declaring an outbreak for all asymptomatic residents (regardless of vaccination status) and all unvaccinated staff. 30,35 Chemoprophylaxis should be continued for 10 days or until the outbreak is over, whichever is longer. ${ }^{35}$ There may be a role to extend this approach of antiviral 'ring prophylaxis' in other closed or semi-closed environments (i.e. cruise ships, military barracks, boarding schools) where antiviral prophylaxis in close contacts may truncate the spread of infection. ${ }^{35,36}$

Antiviral prophylaxis has also been shown to be effective in inpatient settings, particularly for immunocompromised patients. ${ }^{37}$

\section{Limitations of influenza prophylaxis}

Chemoprophylaxis does not completely eliminate the risk of influenza and susceptibility to infection returns once antiviral prophylaxis is stopped..$^{29}$ In an outbreak, neuraminidase inhibitors may be ineffective at preventing asymptomatic influenza (meaning transmission may still occur). In the long term, chemoprophylaxis may result in the emergence of influenza viruses with reduced susceptibility to these drugs. ${ }^{22,23,32}$

\section{Novel therapies}

Several novel therapies are being developed for influenza treatment. These target various stages of influenza infection including prevention of viral entry (DAS181-F03), fusion with host cells (Arbidol), viral transcription and replication (Favipiravir, Pimodovir, S 033188) and maturation of key viral proteins (nitazoxanide). ${ }^{38,39}$ There are also several monoclonal antibodies being developed that target viral structures, primarily haemagglutinin, to neutralise the virus. ${ }^{38}$ Combination therapy with oseltamivir, amantadine, and ribavirin has also been studied but has shown no clinical benefit over oseltamivir alone. ${ }^{40}$

\section{Conclusion}

Influenza infection is an important public health problem, with a substantial disease burden in Australia and worldwide. Vaccination is the most important tool in influenza prevention. Current antiviral therapies have a modest effect on symptom duration with no effect on viral shedding or disease complications. Ongoing research is required to develop more effective therapies and combat emerging antiviral resistance. $\varangle$

\section{REFERENCES}

1. Greenberg DP, Robertson CA, Landolfi VA, Bhaumik A, Senders SD, Decker MD. Safety and immunogenicity of an inactivated quadrivalent influenza vaccine in children 6 months through 8 years of age. Pediatr Infect Dis J 2014;33:630-6. https://doi.org/10.1097/ INF.0000000000000254

2. Kieninger D, Sheldon E, Lin WY, Yu CJ, Bayas JM, Gabor JJ, et al. Immunogenicity, reactogenicity and safety of an inactivated quadrivalent influenza vaccine candidate versus inactivated trivalent influenza vaccine: a phase III, randomized trial in adults aged $\geq 18$ years. BMC Infect Dis 2013;13:343. https://doi.org/10.1186/1471-2334-13-343

3. van de Witte S, Nauta J, Montomoli E, Weckx J. A phase III randomised trial of the immunogenicity and safety of quadrivalent versus trivalent inactivated subunit influenza vaccine in adult and elderly subjects, assessing both anti-haemagglutinin and virus neutralisation antibody responses. Vaccine 2018;36:6030-8. https://doi.org/10.1016/j.vaccine.2018.04.043

4. Treanor JT, Albano FR, Sawlwin DC, Graves Jones A, Airey J, Formica N, et al. Immunogenicity and safety of a quadrivalent inactivated influenza vaccine compared with two trivalent inactivated influenza vaccines containing alternate B strains in adults: a phase 3, randomized noninferiority study. Vaccine 2017:35:1856-64. https://doi.org/10.1016/j.vaccine.2017.02.066

5. DiazGranados CA, Dunning AJ, Kimmel M, Kirby D, Treanor J, Collins A, et al. Efficacy of high-dose versus standard-dose influenza vaccine in older adults. N Engl J Med 2014;371:635-45. https://doi.org/10.1056/NEJMoa1315727

6. Frey SE, Reyes MR, Reynales H, Bermal NN, Nicolay U, Narasimhan V, et al. Comparison of the safety and immunogenicity of an MF59 ${ }^{\circledR}$-adjuvanted with a non-adjuvanted seasonal influenza vaccine in elderly subjects. Vaccine 2014;32:5027-34. https://doi.org/10.1016/j.vaccine.2014.07.013
7. Domnich A, Arata L, Amicizia D, Puig-Barberà J, Gasparini R, Panatto D. Effectiveness of MF59-adjuvanted seasonal influenza vaccine in the elderly: a systematic review and meta-analysis. Vaccine 2017;35:513-20. https://doi.org/10.1016/j.vaccine.2016.12.011

8. Australian Government Department of Health, Statement from the Chief Medical Officer. 2019 influenza vaccines [Internet]. https://beta.health.gov.au/ news-and-events/media-releases/2019-influenza-vaccines [cited 2019 Mar 14]

9. Greenberg DP, Robertson CA, Noss MJ, Blatter MM, Biedenbender R, Decker MD. Safety and immunogenicity of a quadrivalent inactivated influenza vaccine compared to licensed trivalent inactivated influenza vaccines in adults. Vaccine 2013;31:770-6. https://doi.org/10.1016/ j.vaccine.2012.11.074

10. Thompson WW, Shay DK, Weintraub E, Brammer L, Bridges CB, Cox NJ, et al. Influenza-associated hospitalizations in the United States. JAMA 2004;292:1333-40. https://doi.org/10.1001/jama.292.11.1333

11. Sandoval C, Walter SD, Krueger P, Smieja M, Smith A, Yusuf S, et al. Risk of hospitalization during influenza season among a cohort of patients with congestive heart failure. Epidemiol Infect 2007;135:574-82. https://doi.org/ 10.1017/S095026880600714X

12. Natori Y, Shiotsuka M, Slomovic J, Hoschler K, Ferreira V, Ashton P, et al. A double-blind, randomized trial of high-dose vs standard-dose influenza vaccine in adult solid-organ transplant recipients. Clin Infect Dis 2018;66:1698-704. https://doi.org/10.1093/cid/cix1082

13. Mannino S, Villa M, Apolone G, Weiss NS, Groth N, Aquino I, et al. Effectiveness of adjuvanted influenza vaccination in elderly subjects in northern Italy. Am J Epidemiol 2012;176:527-33. https://doi.org/10.1093/aje/ kws313 
14. Izurieta HS, Thadani N, Shay DK, Lu Y, Maurer A, Foppa IM, et al. Comparative effectiveness of high-dose versus standard-dose influenza vaccines in US residents aged 65 years and older from 2012 to 2013 using Medicare data: a retrospective cohort analysis. Lancet Infect Dis 2015;15:293-300. https://doi.org/10.1016/S1473-3099(14)71087-4

15. Gravenstein S, Davidson HE, Taljaard M, Ogarek J, Gozalo P, Han L, et al Comparative effectiveness of high-dose versus standard-dose influenza vaccination on numbers of US nursing home residents admitted to hospital: a cluster-randomised trial. Lancet Respir Med 2017;5:738-46. https://doi.org/ 10.1016/S2213-2600(17)30235-7

16. Shay DK, Chillarige Y, Kelman J, Forshee RA, Foppa IM, Wernecke M, et al. Comparative effectiveness of high-dose versus standard-dose influenza vaccines among US Medicare beneficiaries in preventing postinfluenza deaths during 2012-2013 and 2013-2014. J Infect Dis 2017:215:510-7. https://doi.org/ 10.1093/infdis/jiw641

17. Kaka AS, Filice GA, Myllenbeck S, Nichol KL. Comparison of side effects of the 2015-2016 high-dose, inactivated, trivalent influenza vaccine and standard dose, inactivated, trivalent influenza vaccine in adults $\geq 65$ years Open Forum Infect Dis 2017:4:ofx001. https://doi.org/10.1093/ofid/ofx001

18. Villa M, Black S, Groth N, Rothman KJ, Apolone G, Weiss NS, et al. Safety of MF59-adjuvanted influenza vaccination in the elderly: results of a comparative study of MF59-adjuvanted vaccine versus nonadjuvanted influenza vaccine in northern Italy. Am J Epidemiol 2013;178:1139-45. https://doi.org/10.1093/aje/ kwt078

19. Tricco AC, Chit A, Soobiah C, Hallett D, Meier G, Chen MH, et al Comparing influenza vaccine efficacy against mismatched and matched strains: a systematic review and meta-analysis. BMC Med 2013;11:153. https://doi.org/ 10.1186/1741-7015-11-153

20. Bennett JE, Dolin R, Blaser MJ. Mandell, Douglas, and Bennett's principles and practices of infectious diseases. 8th ed. Philadelphia, PA: Elsevier; 2015.

21. Therapeutic Goods Administration. Australian Register of Therapeutic Goods [Internet]. www.tga.gov.au/artg [cited 2019 Mar 1]

22. Jefferson T, Jones MA, Doshi P, Del Mar CB, Hama R, Thompson MJ, et al. Neuraminidase inhibitors for preventing and treating influenza in healthy adults and children. Cochrane Database Syst Rev 2014:CD008965. https://doi.org/10.1002/14651858.CD008965.pub4

23. Heneghan CJ, Onakpoya I, Jones MA, Doshi P, Del Mar CB, Hama R, et al. Neuraminidase inhibitors for influenza: a systematic review and meta-analysis of regulatory and mortality data. Health Technol Assess 2016;20:1-242. https://doi.org/10.3310/hta20420

24. Kohno S, Yen MY, Cheong HJ, Hirotsu N, Ishida T, Kadota J, et al.; S-021812 Clinical Study Group. Phase III randomized, double-blind study comparing single-dose intravenous peramivir with oral oseltamivir in patients with seasonal influenza virus infection. Antimicrob Agents Chemother 2011;55:5267-76. https://doi.org/10.1128/AAC.00360-11

25. Koyama K, Takahashi M, Oitate M, Nakai N, Takakusa H, Miura S, et al. CS-8958, a prodrug of the novel neuraminidase inhibitor R-125489, demonstrates a favorable long-retention profile in the mouse respiratory tract. Antimicrob Agents Chemother 2009;53:4845-51. https://doi.org/ 10.1128/AAC.00731-09

26. Watanabe A, Chang SC, Kim MJ, Chu DW, Ohashi Y; MARVEL Study Group. Long-acting neuraminidase inhibitor laninamivir octanoate versus oseltamivir for treatment of influenza: a double-blind, randomized, noninferiority clinical trial. Clin Infect Dis 2010;51:1167-75. https://doi.org/10.1086/656802

27. Marty FM, Vidal-Puigserver J, Clark C, Gupta SK, Merino E, Garot D, et al. Intravenous zanamivir or oral oseltamivir for hospitalised patients with influenza: an international, randomised, double-blind, double-dummy, phase 3 trial. Lancet Respir Med 2017;5:135-46. https://doi.org/10.1016/ S2213-2600(16)30435-0
28. Muthuri SG, Venkatesan S, Myles PR, Leonardi-Bee J, Al Khuwaitir TS, Al Mamun A, et al.; PRIDE Consortium Investigators. Effectiveness of neuraminidase inhibitors in reducing mortality in patients admitted to hospita with influenza A H1N1pdm09 virus infection: a meta-analysis of individual participant data. Lancet Respir Med 2014;2:395-404. https://doi.org/10.1016/ S2213-2600(14)70041-4

29. Fiore AE, Fry A, Shay D, Gubareva L, Bresee JS, Uyeki TM; Centers for Disease Control and Prevention (CDC). Antiviral agents for the treatment and chemoprophylaxis of influenza --- recommendations of the Advisory Committee on Immunization Practices (ACIP). MMWR Recomm Rep 2011;60:1-24.

30. Harper SA, Bradley JS, Englund JA, File TM, Gravenstein S, Hayden FG, et al.; Expert Panel of the Infectious Diseases Society of America. Seasonal influenza in adults and children--diagnosis, treatment, chemoprophylaxis, and institutional outbreak management: clinical practice guidelines of the Infectious Diseases Society of America. Clin Infect Dis 2009;48:1003-32. https://doi.org/10.1086/598513

31. Antibiotic. In ETG complete [Internet]. Melbourne: Therapeutic Guidelines Limited; 2019. www.tg.org.au [cited 2019 Mar 1]

32. Li TC, Chan MC, Lee N. Clinical implications of antiviral resistance in influenza. Viruses 2015;7:4929-44. https://doi.org/10.3390/v7092850

33. Moore C, Galiano M, Lackenby A, Abdelrahman T, Barnes R, Evans MR, et al. Evidence of person-to-person transmission of oseltamivir-resistant pandemic influenza A(H1N1) 2009 virus in a hematology unit. J Infect Dis 2011;203:18-24. https://doi.org/10.1093/infdis/jiq007

34. Jefferson T, Demicheli V, Di Pietrantonj C, Rivetti D. Amantadine and rimantadine for influenza A in adults. Cochrane Database Syst Rev 2006:CD001169. https://doi.org/10.1002/14651858.CD001169.pub3

35. Communicable Diseases Network of Australia (CDNA). Guidelines for the prevention, control and public health management of influenza outbreaks in residential care facilities in Australia [Internet]. Canberra: Department of Health; 2017. www.health.gov.au/internet/main/publishing.nsf/Content/ cdna-flu-guidelines.htm [cited 2019 Mar 1]

36. Lee VJ, Yap J, Cook AR, Chen MI, Tay JK, Tan BH, et al. Oseltamivir ring prophylaxis for containment of 2009 H1N1 influenza outbreaks. N Engl J Med 2010;362:2166-74. https://doi.org/10.1056/NEJMoa0908482

37. Yue MC, Collins JT, Subramoniapillai E, Kennedy GA. Successful use of oseltamivir prophylaxis in managing a nosocomial outbreak of influenza $A$ in a hematology and allogeneic stem cell transplant unit. Asia Pac J Clin Oncol 2017;13:37-43. https://doi.org/10.1111/ajco.12565

38. Koszalka P, Tilmanis D, Hurt AC. Influenza antivirals currently in late-phase clinical trial. Influenza Other Respir Viruses 2017;11:240-6. https://doi.org/ 10.1111/irv.12446

39. Boriskin YS, Leneva IA, Pecheur El, Polyak SJ, et al. Arbidol: a broad-spectrum antiviral compound that blocks viral fusion. Curr Med Chem 2008:15:997-1005. https://doi.org/10.2174/092986708784049658

40. Beigel JH, Bao Y, Beeler J, Manosuthi W, Slandzicki A, Dar SM, et al.; IRC003 Study Team. Oseltamivir, amantadine, and ribavirin combination antiviral therapy versus oseltamivir monotherapy for the treatment of influenza: a multicentre, double-blind, randomised phase 2 trial. Lancet Infect Dis 2017:17:1255-65. https://doi.org/10.1016/S1473-3099(17)30476-0

\section{FURTHER READING}

Australian Technical Advisory Group on Immunisation. Australian immunisation handbook [Internet]. Canberra: Department of Health; 2018.

https://immunisationhandbook.health.gov.au [cited 2019 Mar 1]
Foo H, Dwyer DE. Rapid tests for the diagnosis of influenza. Aust Prescr 2009;32:64-7. https://doi.org/10.18773/austprescr.2009.034 